Supporting information for:

\title{
Auranofin-Based Analogues Are Effective Against Clear Cell Renal Carcinoma in vivo and Display No Significant Systemic Toxicity
}

Benelita T. Elie, ${ }^{a, b}$ Karen Hubbard, ${ }^{b, e}$ Buddhadev Layek, ${ }^{f}$ Won Seok Yang, ${ }^{g}$ Swayam Prabha, ${ }^{f}$ Joe W. Ramos, ${ }^{g, *}$ and Maria Contel ${ }^{a, b-d, g, *}$

${ }^{a}$ Department of Chemistry, Brooklyn College, The City University of New York; Brooklyn, New York; ${ }^{b}$ Biology, ${ }^{\mathrm{c} C h e m i s t r y}$ and ${ }^{\mathrm{d}}$ Biochemistry PhD Programs, The Graduate Center, The City

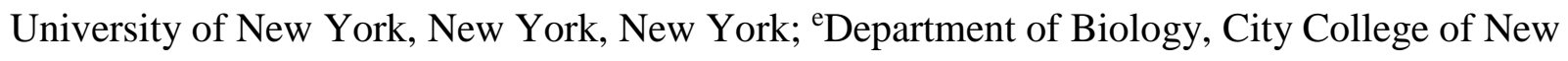

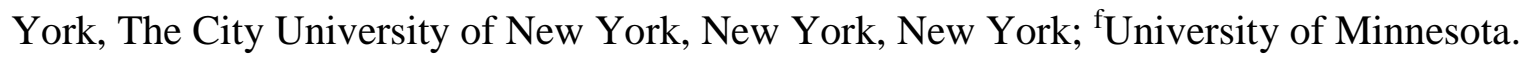
College of Pharmacy, Minneapolis, Minnesota; ${ }^{\circ}$ University of Hawaii Cancer Center, Honolulu, Hawaii.

This PDF includes Supplementary Tables 1, 2 and 3 related to the pathology study. 
Table S1. Summary of plasma metabolic analytes for specimens from tumor bearing NOD-SCID (NOD/LtSz-Prkdscid/j) mice. The study criteria reflect pathological criterion used in clinical studies. Metabolic markers were quantified from freshly isolated plasma and compared to reference range. Titanocref: 3 specimens, at $5 \mathrm{mg} / \mathrm{kg} / 72$ hours over 21 days. Titanofin: 2 specimens, at $10 \mathrm{mg} / \mathrm{kg} / 72$ hours over 21 days.

\section{BUN (mg/dL)}

CREA (mg/dL)

BUN/CREA ratio

$\operatorname{ALP}(\mathbf{U} / \mathbf{L})$

$\operatorname{ALT}(\mathbf{U} / \mathbf{L})$

$\operatorname{AST}(\mathbf{U} / \mathbf{L})$

GGT (U/L)

TBIL (mg/dL)

DBIL (mg/dL)

$\operatorname{IBIL}(\mathrm{mg} / \mathrm{dL})$

TP $(\mathbf{g} / \mathbf{d L})$

ALB (g/dL)

GLOB (g/dL)

A/G ratio

P (mg/dL)

$\mathrm{Ca}$ (mg/dL)

GLU (mg/dL)

CHOL (mg/dL)

TRIG (mg/dL)

CK (U/L)

TCO2 (mEq/L)

$\mathrm{Na}(\mathrm{mEq} / \mathrm{L})$

$\mathrm{K}(\mathrm{mEq} / \mathrm{L})$

$\mathrm{Cl}(\mathrm{mEq} / \mathrm{L})$

$\mathrm{Na} / \mathrm{K}$ ratio

Anion Gap

\begin{tabular}{c} 
Titanocref \\
\hline 22.33 \\
0.17 \\
$128.99 * \boldsymbol{\nabla}$ \\
$37 * \boldsymbol{\nabla}$ \\
$36.33 * \nabla$ \\
$135.67 * \nabla \bigcirc$ \\
0.00 \\
0.17 \\
0.03 \\
0.13 \\
5.03
\end{tabular}

2.70

2.33

1.17

8.87

9.33

$166.33 *$ v

85.00

$109 * \Delta$

$$
278.67 * \nabla(0)
$$

22.67

157.00

8.13

110.67

19.66

31.80

\section{Titanofin}

21.50

0.17

$126.47 * \nabla$

$42.5 * \nabla$

$167 *$ v

$374.5 * \nabla(0)$

0.00

0.20

0.05

0.15

$5.3^{* \Delta}$

2.95

2.35

1.26

9.95

9.60

$170 * \boldsymbol{v}$

88.00

76.50

$196.5^{*}$ จ (

21.50

155.00

9.30

112.00

16.74

$30.8 * \boldsymbol{\nabla}$

Vehicle

21.50

0.16

142.50

55.50

199.00

$402.50 \bigcirc$

0.00

0.20

0.00

0.20

4.95

2.80

2.15

1.30

9.80

9.20

189.50

85.50

74.00

566.50

18.00

156.00

10.00

115.00

16.24

33.00
Reference Ranges

5.0-28

$0.2-0.5$

105-370

27-195

54-77

$0.2-0.6$

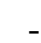

$-$

4.8-7.2

$2.4-4.3$

$1.7-2.2$

7.3-14.5

9.5-12.5

172-372

55-169

67-289

428-1609

145-181

7.3-11.1

111-134

$* \mathrm{P}<0.05$, values statistically different between Vehicle-treated and Titanocref-treated or Titanofin-treated groups. $\checkmark$ Titanocref or Titanofin induce a decrease from Veh treated group, $\bullet$ Titanocref or Titanofin induce an increase from Veh treated group, () deviates significantly from the normal range.

ALP, alakaline phosphatase; AST, aspartate aminotransferase; Ca, calcium; $\mathrm{Cl}$, chloride; $\mathrm{CPK}$, creatine phosphokinase; $\mathrm{P}$, phosphorus; K, potassium; Na, sodium; BUN, blood urea nitrogen; Statistical significance $(* \mathrm{P}<0.05)$. 
Table S2. Summary of hematology values (mean) for specimens from NOD-SCID (NOD/LtSzPrkdscid/j) mice. Complete blood counts of each group. Heparinized blood acquired from mice was assessed using the automatic Hematology Analyzer. Blood smears were carried out and fixed with methanol, stained, and observed under the microscope to carry out differential blood white cell counts. Titanocref: 3 specimens, at $5 \mathrm{mg} / \mathrm{kg} / 72$ hours over 21 days. Titanofin: 2 specimens, at $10 \mathrm{mg} / \mathrm{kg} / 72$ hours over 21 days.

\section{Automatic count}

\begin{tabular}{|c|c|c|c|c|}
\hline & Titanocref & Titanofin & Vehicle & NSG Reference Range \\
\hline $\mathrm{RBC}(\mathrm{M} / \mathrm{uL})$ & $9.02 * \Delta$ & $9.375 * \Delta$ & 7.415 & $7.84-10.84$ \\
\hline $\mathrm{HGB}(\mathrm{g} / \mathrm{dL})$ & $13.83 * \Delta$ & $14.3 * \Delta$ & 11.45 & $11.8-17.6$ \\
\hline $\operatorname{HCT}(\%)$ & $45.67 * \Delta$ & $48.35^{* \boldsymbol{\Lambda}}$ & 36.9 & $44.1-58.3$ \\
\hline MCV (fL) & 50.67 & 51.55 & 49.1 & $51.1-58.6$ \\
\hline $\mathrm{MCH}(\mathrm{pg})$ & 15.33 & 15.25 & 15.5 & $13.7-17.2$ \\
\hline MCHC (g/dL) & 30.30 & 29.6 & 31.7 & $25.1-31.3$ \\
\hline RDW-SD (fL) & 29.23 & 30.65 & 29.35 & - \\
\hline RDW-CV (\%) & 22.23 & 22.5 & 20.5 & $17.3-20.3$ \\
\hline RET\# (K/uL) & $318.47 * \nabla$ & 427.55 & 444.4 & $294-444$ \\
\hline RET (\%) & 3.52 & 4.56 & 4.62 & $2.56-4.56$ \\
\hline PLT (K/uL) & $1244.00 * \Delta$ & $1327.5 * \Delta$ & 712.5 & $651-2055$ \\
\hline PDW (fL) & 6.67 & 6.65 & 6.5 & - \\
\hline MPV (fL) & 6.1 & 6.05 & 5.9 & $4.2-6.3$ \\
\hline Morphology & $\begin{array}{l}\text { 1+ Echinocytes. 3+ } \\
\text { polychromasia. } 2+ \\
\text { HGB Crystals. 2+ } \\
\text { polychromasia. } 3+ \\
\text { PLT clumps. }\end{array}$ & $\begin{array}{c}2+\text { polychromasia. } \\
\text { A manual } \\
\text { differential was } \\
\text { not performed due } \\
\text { to low } \mathrm{WBC} \text { count } \\
(<2.00 \mathrm{~K} / \mathrm{uL}) \text {. }\end{array}$ & $\begin{array}{c}2+ \\
\text { polychromasia. }\end{array}$ & \\
\hline
\end{tabular}




\section{Manual differential}

Titanocref

Neut\# (K/uL)
Band\# (K/uL)
LYMPH\# (K/uL
MONO\# (K/uL)
EO\# (K/uL)
NEUT (\%)
LYMPH (\%)
MONO (\%)
EO (\%)

Morphology

$\begin{array}{cc}2.21 * \boldsymbol{\Delta} & 0.845 * \boldsymbol{\nabla} \\ 0.00 & 0 \\ 0.85 * \boldsymbol{\Delta} & 0.38 \\ 0.00 & 0 \\ 0.02 * \boldsymbol{\nabla} & 0.125 * \boldsymbol{\Delta} \\ 68.33 & 62 * \boldsymbol{\nabla} \\ 26.33 * \boldsymbol{\Delta} & 28 * \boldsymbol{\Delta} \\ 4.50 * \mathbf{\Delta} & 5 * \boldsymbol{\Delta} \\ 3.50 & 5 * \boldsymbol{\Delta}\end{array}$

WBC morphology is within normal limits.
WBC morphology is within normal limits.
Vehicle

1.675

0.01

0.41

0

0.075

75.5

18.5

2.5

3
NSG Reference Range 0.54-3.16

0.23-1.56

0.03-0.26

0.00-0.39

44.21-79.92

13.51-42.61

1.71-10.93

$0.29-10.32$

\section{WBC}

morphology is within normal

limits.

$* \mathrm{P}<0.05$, values statistically different between Vehicle-treated and Titanocref-treated or Titanofin-treated groups. $\checkmark$ Titanocref or Titanofin induce a decrease from Veh treated group, $\Delta$ Titanocref or Titanofin induce an increase from Veh treated group.

WBC: white blood cells, RBC: red blood cells, Hgb: hemoglobin, HCT: hematocrit, MCV: mean cell volume, $\mathrm{MCH}$ : mean corpuscular hemoglobin content, MCHC: mean corpuscular hemoglobin concentration, RDW-CV: red cell distribution width-coefficient of variation, PLT: platelets, MPV: mean platelet volume. 
Table S3. Histology. Summary of Histopathological Findings.

Body weight (g)

Liver weight (g)

Spleen weight (g)

Heart weight (g)

Left Kidney weight (g)

Right Kidney weight (g)

Heart

Lungs

Thymus

Kidneys

Liver

Gallbladder

Stomach

Duodenum, jejunum, ileum

Cecum

Colon

Mesenteric lymph node

Salivary glands

Submandibular lymph

node

Uterus

Cervix

Vagina

Testes/epididymis

Prostate

Seminal vesicles

Urinary bladder

Spleen

Pancreas
Titanocref

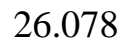

1.363

0.040

0.203

0.225

0.250

$\mathrm{N}$

$\mathrm{N}$

U

$\mathrm{N}$

$\mathrm{N}$

$\mathrm{N}$

$\mathrm{N}$

$\mathrm{N}$

Lymphoplasmacytic and neutrohpilic aggregates,

submucosal, F, 2.

$\mathrm{N}$

$\mathrm{N}$

$\mathrm{N}$

$\mathrm{N}$

$\mathrm{N}$

$\mathrm{N}$

$\mathrm{N}$

Testicular interstitial edema, BL, D, 2.

$\mathrm{N}$

$\mathrm{N}$

$\mathrm{N}$

Increased extramedullary hematopoiesis, 2

$\mathrm{N}$
Titanofin

20.887

1.147

0.048

0.148

0.199

0.168

$\mathrm{N}$

Alveolar histiocytosis, MF, 2.

$\begin{array}{ll}\mathrm{N} & \mathrm{N} \\ \mathrm{N} & \mathrm{N} \\ \mathrm{N} & \mathrm{N} \\ \mathrm{N} & \mathrm{N} \\ \mathrm{N} & \mathrm{N} \\ \mathrm{N} & \mathrm{N}\end{array}$

$\mathrm{N}$

$\mathrm{N}$

$\mathrm{N}$

$\mathrm{N}$

$\mathrm{N}$

$\mathrm{N}$

$\mathrm{N}$

$\mathrm{N}$

$\mathrm{N}$

Lymphoplasmacytic and neutrohpilic aggregates,

submucosal, F, 2.

$$
\begin{aligned}
& \mathrm{N} \\
& \mathrm{N} \\
& \mathrm{N} \\
& \mathrm{N}
\end{aligned}
$$

Cystic endometrial hyperplasia, MF, 2.
$\mathrm{N}$
$\mathrm{N}$
$\mathrm{N}$
$\mathrm{N}$
$\mathrm{N}$

$\mathrm{N}$

Increased

extramedullary

hematopoiesis, 2

$\mathrm{N}$
Increased extramedullary hematopoiesis, 2 Interstitial edema, D, 2. 


\begin{tabular}{|c|c|c|c|}
\hline Adrenals & $\mathrm{N}$ & $\mathrm{N}$ & $\mathrm{N}$ \\
\hline Ovaries & $\mathrm{N}$ & $\mathrm{N}$ & $\mathrm{N}$ \\
\hline Oviducts & $\mathrm{N}$ & $\mathrm{N}$ & $\mathrm{N}$ \\
\hline Trachea & $\mathrm{N}$ & $\mathrm{N}$ & $\mathrm{N}$ \\
\hline Esophagus & $\mathrm{N}$ & $\mathrm{N}$ & $\mathrm{N}$ \\
\hline Thyroid & $\mathrm{N}$ & $\mathrm{N}$ & $\mathrm{N}$ \\
\hline Parathyroid & $\mathrm{U}$ & $\mathrm{U}$ & $\mathrm{U}$ \\
\hline Skin (trunk) & $\begin{array}{l}\text { Mass } 1 \text { and 2: Necrotic } \\
\text { debris and suppurative } \\
\text { inflammation, with } \\
\text { eosinophilic flaky } \\
\text { birefringent material } \\
\text { and fibrovascular } \\
\text { stroma, F, } 4\end{array}$ & $\begin{array}{l}\text { Mass 1: Anaplastic } \\
\text { neoplasm; Mass 2: } \\
\text { Necrotic debris with } \\
\text { eosinophilic flaky } \\
\text { birefringent material, } 2\end{array}$ & $\begin{array}{c}\text { Mass } 1 \text { and 2: } \\
\text { Multifocal anaplastic } \\
\text { neoplasm; Mass 3: } \\
\text { Eosinophilic flaky } \\
\text { birefringent material } \\
\text { with rare necrotic } \\
\text { debris, F, } 2\end{array}$ \\
\hline Mammary glands & NA & $\mathrm{N}$ & $\mathrm{N}$ \\
\hline $\begin{array}{l}\text { Bones (femur, tibia, } \\
\text { sternum, vertebrae) }\end{array}$ & $\mathrm{N}$ & $\mathrm{N}$ & $\mathrm{N}$ \\
\hline $\begin{array}{l}\text { Bone marrow (femur, } \\
\text { tibia, sternum, } \\
\text { vertebrae) }\end{array}$ & $\begin{array}{c}\text { Granulocytic } \\
\text { hyperplasia, D, 2; } \\
\text { Erythroid hypoplasia; } \\
\text { D, } 2 .\end{array}$ & $\mathrm{N}$ & $\mathrm{N}$ \\
\hline Stifle joint & $\mathrm{N}$ & $\mathrm{N}$ & $\mathrm{N}$ \\
\hline $\begin{array}{l}\text { Skeletral muscles (hind } \\
\text { limb, spine) }\end{array}$ & $\mathrm{N}$ & $\mathrm{N}$ & $\mathrm{N}$ \\
\hline $\begin{array}{l}\text { Nerves (hind limb, } \\
\text { spine) }\end{array}$ & $\mathrm{N}$ & $\mathrm{N}$ & $\mathrm{N}$ \\
\hline Spinal cord & $\mathrm{N}$ & $\mathrm{N}$ & $\mathrm{N}$ \\
\hline Oral cavity & $\mathrm{N}$ & $\mathrm{N}$ & $\mathrm{N}$ \\
\hline Teeth & $\mathrm{N}$ & $\mathrm{N}$ & $\mathrm{N}$ \\
\hline Nasal cavity & $\mathrm{N}$ & $\mathrm{N}$ & $\mathrm{N}$ \\
\hline Eyes & $\mathrm{N}$ & $\mathrm{N}$ & $\mathrm{N}$ \\
\hline Harderian gland & $\mathrm{N}$ & $\mathrm{N}$ & $\mathrm{N}$ \\
\hline Bones (skull) & $\mathrm{N}$ & $\mathrm{N}$ & $\mathrm{N}$ \\
\hline Pituitary & $\mathrm{N}$ & $\mathrm{N}$ & $\mathrm{N}$ \\
\hline Brain & $\mathrm{N}$ & $\mathrm{N}$ & $\mathrm{N}$ \\
\hline Ears & $\mathrm{N}$ & $\mathrm{N}$ & $\mathrm{N}$ \\
\hline Other organs & $\mathrm{N}$ & $\mathrm{N}$ & $\mathrm{N}$ \\
\hline \multicolumn{4}{|l|}{$\mathrm{N}$ : Normal } \\
\hline \multicolumn{4}{|l|}{ F: Focal } \\
\hline \multicolumn{4}{|l|}{ MF: Multifocal } \\
\hline \multicolumn{4}{|l|}{ D: Diffuse } \\
\hline \multicolumn{4}{|l|}{ BL: Bilateral } \\
\hline \multicolumn{4}{|l|}{ 1: Minimal } \\
\hline 2: Mild & & & \\
\hline
\end{tabular}


3: Moderate

4: Marked

U: Unavailable 